\title{
Calcium supplement and bone loss
}

\author{
T. V. Nguyen
}

Received: 24 August 2009 / Accepted: 9 September 2009 / Published online: 3 October 2009

(C) International Osteoporosis Foundation and National Osteoporosis Foundation 2009

Dear Editors,

In postmenopausal women, whether supplementation of calcium reduces bone loss or not is a contentious issue. The latest analysis by Professor Nordin [1] is a valiant effort to resolve the issue. By using a meta-analytic approach, Professor Nordin concludes that daily calcium supplement of $100 \mathrm{mg}$ could protect against bone loss for up to 4 years. This conclusion appears to be based on the mean difference in the rate of change in BMD between the control and treated (calcium supplementation) groups. However, a close reading of the analysis reveals a number of methodological shortcomings that could potentially compromise the author's conclusion.

It is well known that the rate of change in BMD varies remarkably among individuals, with the standard deviation being 2-4 times higher than the average $[2,3]$. This heterogeneity is observed not just in nontreated populations, but also in randomized controlled clinical trials [4], where it ranged between $2.1 \%$ and $5 \%$. However, in the present paper, it is reported that the standard deviation of BMD change was less than $1 \%$ for both control and treated groups.

This low variability is likely due to the way the data from individual studies were analyzed. There are two important sources of variation in the rates of change in BMD: between-study and within-study variation. It is critically important to weight the within study variation, because studies with large variance (i.e. less consistent effect) should have less weight than studies with small

Related correspondence can be found at doi:10.1007/s00198-0091016-9, doi:10.1007/s00198-009-1072-1, and doi:10.1007/s00198009-1074-z.

T. V. Nguyen $(\bowtie)$

Osteoporosis and Bone Biology Program,

Garvan Institute of Medical Research,

384 Victoria Street,

Sydney, New South Wales, Australia

e-mail: t.nguyen@garvan.org.au variance (i.e. more consistent effect). It appears that the author did not consider the within-study variance in the analysis, and this probably explained the unusually low standard deviation reported in the paper.

The average rate of change in BMD was actually derived as the mean of averages of change in BMD from various BMD sites (femoral neck, lumbar spine, metacarpal, distal radius, mid-radius, and even total body BMD) from all 32 studies. It is known that, for example, the rates of change in lumbar spine and femoral neck BMD are very different due to bone remodeling; therefore, averaging the rates of change in BMD for the two sites can yield a result that is very difficult to interpret. Moreover, since the BMD values measured at different sites are likely to be correlated, this average approach is not optimal for estimating the "true" rate of BMD change.

The difference in the rate of BMD change between the calcium supplementation and control groups was modest [1], and the statistical significance was achieved due primarily to the accumulative large sample size and the absence of withinstudy variance in the analysis. If the within-study variance had been taken into account, the conclusion of the effect of calcium supplement on bone loss might have been different.

\section{References}

1. Nordin BE (2009) The effect of calcium supplementation on bone loss in 32 controlled trials in postmenopausal women. Osteoporos Int. doi:10.1007/s00198-009-0926-x

2. Jones G, Nguyen T, Sambrook P, Kelly PJ, Eisman JA (1994) Progressive loss of bone in the femoral neck in elderly people: longitudinal findings from the Dubbo osteoporosis epidemiology study. Br Med J 309:691-5

3. Nguyen TV, Pocock N, Eisman JA (2000) Interpretation of bone mineral density measurement and its change. J Clin Densitom 3:107-19

4. Hosking D, Chilvers C, Christiansen C, Ravn P, Wasnich R, Ross P, McClung M, Balke A, Thompson D, Daley M, Yates J (1998) Prevention of bone loss with alendronate in postmenopausal women under 60 years of age. N Engl J Med 338:485-92 